\title{
Cross sections of light-ion reactions calculated from $a b$ initio wave functions
}

\author{
Christian Forssén ${ }^{a} \dagger$, Petr Navrátil ${ }^{a}$, W. Erich Ormand ${ }^{a}$, and Etienne Caurier ${ }^{b}{ }^{\ddagger}$ \\ ${ }^{a}$ Lawrence Livermore National Laboratory, P.O. Box 808, L-414, Livermore, CA 94551, USA \\ ${ }^{b}$ Institut de Recherches Subatomiques (IN2P3-CNRS-Université Louis Pasteur) \\ Batiment 27/1, 67037 Strasbourg Cedex 2, France \\ E-mail: c.forssenefy.chalmers.se
}

\begin{abstract}
We will discuss present attempts to employ many-body nuclear structure information in nuclear reaction calculations. The foundation of our approach is the ab initio no-core shell model (NCSM), which is a well-established theoretical framework aimed at an exact description of nuclear structure starting from high-precision interactions between the nucleons. We have developed the capability to extract translationally invariant cluster form factors from the NCSM many-body wave functions, and subsequently employ them in cross section calculations. We will show some of our first results from studies of the radiative capture reactions ${ }^{3} \mathrm{He}(\alpha, \gamma){ }^{7} \mathrm{Be},{ }^{7} \mathrm{Be}(p, \gamma){ }^{8} \mathrm{~B}$ and ${ }^{10} \mathrm{Be}(n, \gamma){ }^{11} \mathrm{Be}$. The former two reactions correspond to the most important uncertainties in solar model predictions of neutrino fluxes, while the latter represents a possible breakout from the standard primordial nucleosynthesis in inhomogeneous Big Bang scenarios.
\end{abstract}

International Symposium on Nuclear Astrophysics - Nuclei in the Cosmos - IX

25-30 June 2006

CERN

\footnotetext{
* Speaker.

${ }^{\dagger}$ Present address: Fundamental Physics, Chalmers University of Technology, 41296 Gothenburg, Sweden

This work was partly performed under the auspices of the U. S. Department of Energy by the University of California, Lawrence Livermore National Laboratory, under contract No. W-7405-Eng-48.
} 


\section{Introduction}

The most appropriate theoretical description of nuclear reactions obviously depends on the type of reaction being studied. Low-energy, light-ion reactions are generally very sensitive to details of the nuclear structure. At present, this is the type of reactions that we have some hope of describing from a fundamental approach.

At low energies, the relative motion of two composite nuclei will depend on all $A$-nucleon degrees of freedom. Therefore, one of the foremost tasks of the theory of nucleus-nucleus collisions is to formulate and justify equations of motion for this collective degree of freedom. Two very different approaches can be used: (1) A potential model description with simple local internucleus potentials that may be derived by fitting experimental data or from some semi-microscopically founded formalism; (2) First-principles microscopic approaches based on many-nucleon wave functions and realistic interactions between the nucleons. It is important to realize that these two, seemingly very different approaches, do not have to contradict each other. It should be possible to establish, at least a quantitative, relation between the collective interaction potentials and the matrix elements of the many-body Hamiltonian. The intellectually satisfying fully microscopic approach is obviously hard to apply in all but the simplest cases. On the other hand, the phenomenological approach harbors the danger of introducing too many adjustable parameters so that available data are reproduced nicely, but the predictive power is lost. We would like to claim that the task of developing a microscopic and fundamental approach with truly predictive power is becoming possible. The three main ingredients are all available to us:

1. The huge amount of nucleon-nucleon scattering data has led to the arrival of some very accurate internucleon potentials so that we can construct state-of-the-art nuclear Hamiltonians.

2. A number of sophisticated many-body nuclear structure models have been developed over the recent years.

3. The incredible increase of computing capabilities has allowed previously intractable problems to be studied.

In this paper we will present some of the very first results from a rather ambitious research project initiated at Lawrence Livermore National Laboratory. We are attempting to develop the tools for a fundamental description of nuclear reactions starting from realistic interactions between the nucleons and solving the $A$-body problem as accurately as possible. Our approach is based on the ab initio no-core shell model (NCSM), which is a well-established theoretical framework for studying strongly interacting, quantum many-body systems. This method has been applied with great success to nuclear systems, see e.g. Ref. [1]. It is a matrix diagonalization technique to solve the translational invariant A-body problem in a finite harmonic oscillator basis. The basis truncation is defined by the total number of oscillator quanta excitations $\left(\leq N_{\max } \hbar \Omega\right)$ for the $A$ particles. A key ingredient of the approach is the application of a unitary transformation of the bare Hamiltonian to compute a model-space dependent effective interaction. 


\section{Cluster overlaps from ab initio no-core shell model wave functions}

From here on we will assume that we have done our very best to obtain a realistic $A$-body wave function. We can study the decomposition of this wave function into cluster components by introducing a set of cluster state basis functions. They contain the intrinsic eigenstates of the two clusters as well as an antisymmetrizer taking care of the exchange of nucleons between them. We define the cluster overlap function as being the overlap of these basis states with the full $A$-body state. Note that in general this eigenstate can be either a bound or a scattering state of the $A$-body system. For bound states it makes sense to introduce the so called spectroscopic factor which is just the total norm of the cluster overlap function. This quantity can be thought of as a coefficient of parentage characterizing the weight of a particular cluster configuration in the full $A$-body state.

The formalism and codes that are necessary to calculate these cluster overlaps from our NCSM wave functions was recently developed [2]. In Fig. 1(a) we plot the overlap of the ${ }^{11} \operatorname{Be}\left(1 / 2^{+}\right)$ ground state with the ${ }^{10} \mathrm{Be}$ ground state plus a $s_{1 / 2}$ neutron. The nuclear structure of these nuclei were studied extensively using the NCSM in Ref. [3]. This example illustrates a number of important features of our calculated ab initio cluster overlaps. First of all, the fact that we find a node in this overlap is a direct consequence of the full treatment of the Pauli principle in our approach. Secondly, we note that the calculated overlap is very stable with increasing model space. The amplitude in the interior region decreases slightly and the tail builds up slowly. The net effect is that the spectroscopic factor changes very little; less than $1 \%$ in the example shown here. The final observation is that the calculated overlap does not display the physically correct exponential tail at large interfragment distances. We will come back to this observation in the next section.

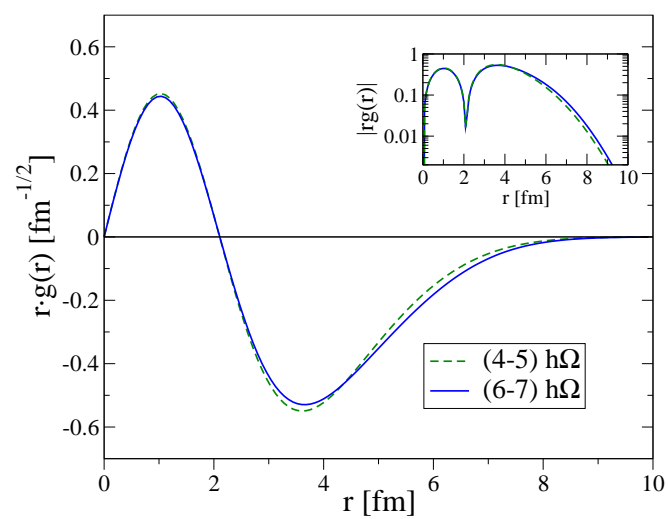

(a)

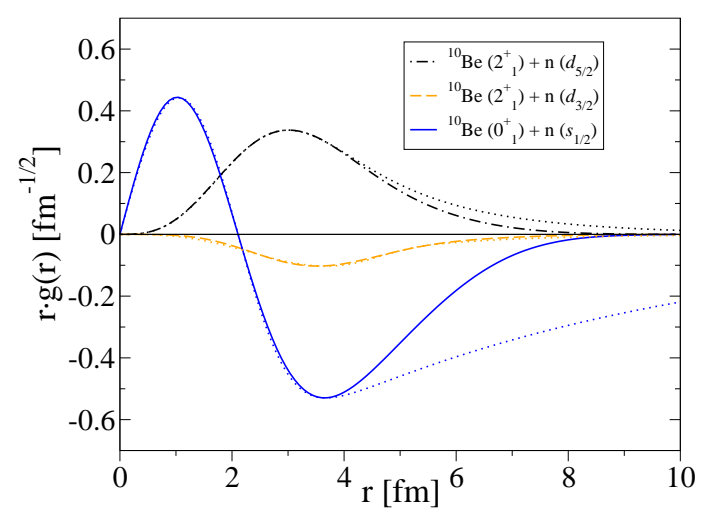

(b)

Figure 1: Cluster overlap functions for ${ }^{11} \mathrm{Be}\left(1 / 2^{+}\right)$with different ${ }^{10} \mathrm{Be}+\mathrm{n}$ channels: (a) ${ }^{10} \mathrm{Be}\left(0_{\mathrm{gs}}^{+}\right)+n\left(s_{1 / 2}\right)$ overlap calculated in two different model spaces; (b) Cluster overlaps for several ${ }^{10} \mathrm{Be}+\mathrm{n}$ channels compared to the fitted Woods-Saxon solutions (dotted lines).

\section{Modeling of direct radiative capture cross sections}

Let us first introduce the connection to the modeling of nuclear reactions. Of great interest in nuclear astrophysics is low-energy, direct radiative capture cross sections. A number of interesting reactions have been discussed at this conference. In first-order perturbation theory, the probability 
for this reaction depends on the matrix element $\left\langle\Phi_{f}\left|H_{\mathrm{int}}\right| \psi_{i}\right\rangle$, where the initial state is a scattering state of a target and a projectile, the final state contains a bound $A$-body state, and the perturbation Hamiltonian describes the interaction between the photon electromagnetic field and the nucleus. How can we utilize our $a b$ initio nuclear structure information in the modeling of this matrix element?

The first step is to compute the wave functions of the final (bound) A-body state, as well as the projectile and target eigenstates with the NCSM. Cluster overlaps are then calculated for a few relevant two-body channels and we remind the reader that the spectroscopic factors equal the integrals of the cluster overlaps. The goal of our project is a fully ab initio approach in the style of the Resonating Group Method. A few more ingredients are still needed and at this stage we are utilizing supplemental input to perform the pilot studies that are shown in this paper. First of all, the dynamics of the scattering wave function is not calculated $a b$ initio but is obtained using an optical potential. In addition, we are correcting our $a b$ initio overlaps to have the physically correct asymptotic behavior. The asymptotic behavior of the interfragment relative motion depends directly on the separation energy for the channel. However, modern nucleon-nucleon interactions don't reproduce binding energies of $A$-body systems with the required precision, even though the inclusion of three-body interactions does improve the situation. In addition, the harmonic oscillator single-particle basis that is employed in the NCSM is not optimized to reproduce exponential tails at very large distances. A temporary fix to this problem can be achieved by constructing so called effective interfragment potentials. We are using simple Woods-Saxon potentials and we are choosing their parameters so that the solution has the best possible fit to our ab initio overlap in the entire interior region; from $0 \mathrm{fm}$ up to $\sim 4 \mathrm{fm}$. At the same time the overall strength is fixed so as to reproduce the experimental separation energy. In Fig. 1(b) we show the results of this procedure for the overlaps of several ${ }^{10} \mathrm{Be}+\mathrm{n}$ channels with the ${ }^{11} \mathrm{Be}\left(1 / 2^{+}\right)$ground state. In the final step, we use our observation that the ab initio spectroscopic factor is well converged and we renormalize our corrected overlap to this value.

\section{1 ${ }^{10} \mathbf{B e}(n, \gamma){ }^{11} \mathbf{B e}$}

It has been suggested that inhomogeneities in the distribution of baryons during the primordial nucleosynthesis can lead to the production of heavy elements [4]. This occurs through a reaction network that consists predominantly of neutron capture reactions. One of the possible breakout chains is limited by the bottleneck reaction. We have studied this reaction using ab initio cluster overlaps of bound states fitted in the 0-4 fm range. The nuclear eigenstates were obtained using the NCSM with the CD-Bonn 2000 interaction in model spaces up to $7 \hbar \Omega$. The effective interfragment potentials that were obtained from the fit were also used to calculate the scattering states. The resulting reaction rate for this reaction is shown in Fig. 2(a), compared to earlier estimates by $\mathrm{T}$. Rauscher et al [4] and A. Mengoni [5]. Our calculations indicate that p-wave capture dominates at the relevant temperatures and that resonant capture is negligible. Further details will be presented in [6]. These results most likely confirm the conclusion that ${ }^{8} \mathrm{Li}(\alpha, n){ }^{11} \mathrm{~B}$ is the relevant bottleneck in the inhomogeneous big bang reaction network; although a full simulation of the entire reaction network has not been performed . 


\section{2 ${ }^{7} \mathbf{B e}(p, \gamma)^{8} \mathbf{B}$}

The ${ }^{7} \mathrm{Be}(p, \gamma)^{8} \mathrm{~B}$ reaction, which is responsible for the total flux of high-energy neutrinos from the sun, was studied in Ref. [7] using the NCSM approach outlined here. Systematic convergence tests, investigating also the dependence on the choice of harmonic oscillator frequency, were performed and resulted in a recommended $S$-factor of $S_{17}=22.1 \pm 1.0 \mathrm{eVb}$. The scattering state was obtained from a phenomenological optical potential that reproduces the position of a ${ }^{8} \mathrm{~B}\left(1^{+}\right)$resonance. The energy dependence of the calculated $S$-factor is shown in Fig. 2(b) compared to data from a number of direct and indirect measurements.

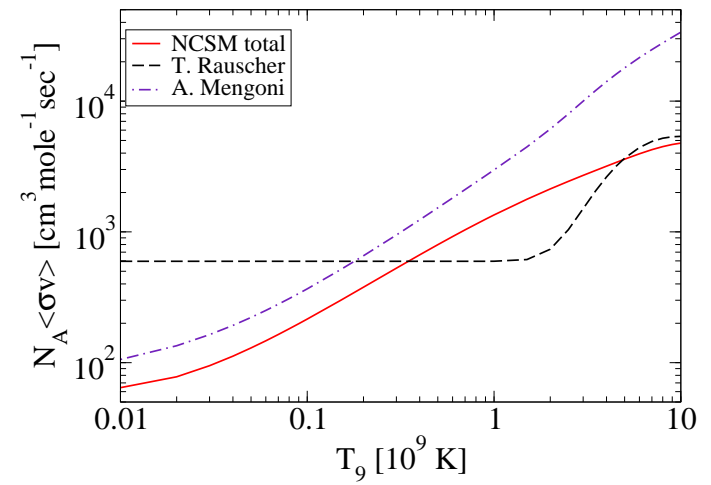

(a)

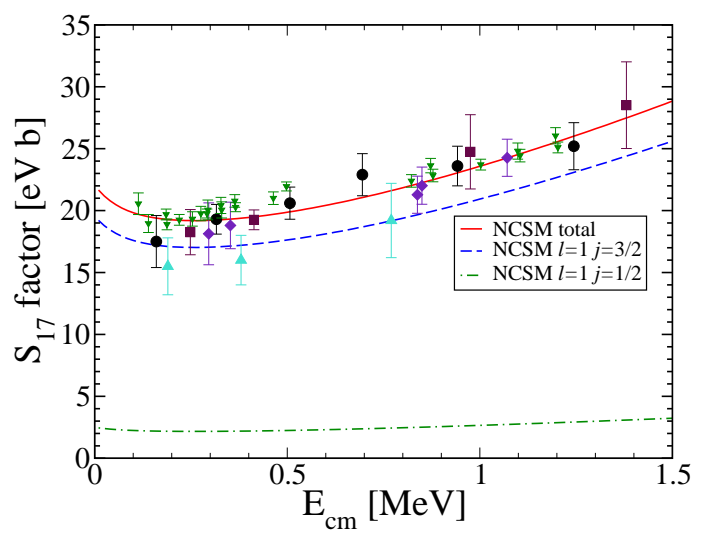

(b)

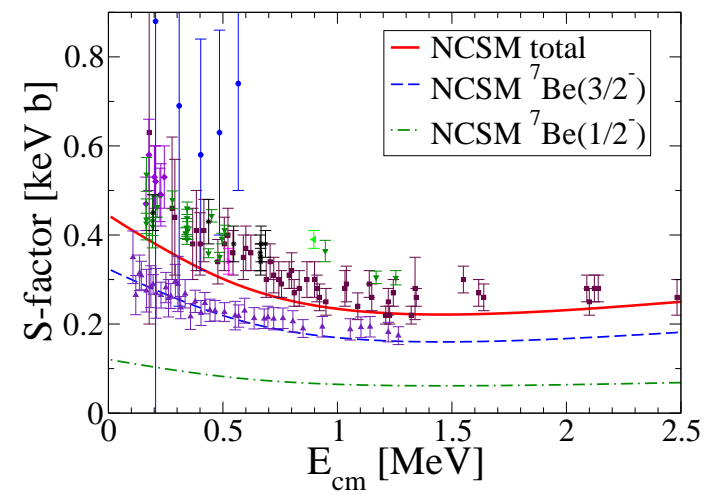

(c)

Figure 2: Selected results for several direct radiative capture reactions compared to available experimental data: (a) Reaction rate for ${ }^{10} \mathrm{Be}(n, \gamma){ }^{11} \mathrm{Be}$ as a function of temperature; (b) $S$-factor for ${ }^{7} \mathrm{Be}(p, \gamma)^{8} \mathrm{~B}$; (c) $S$-factor for ${ }^{3} \mathrm{He}(\alpha, \gamma){ }^{7} \mathrm{Be}$

\section{$3.3{ }^{3} \mathrm{He}(\alpha, \gamma){ }^{7} \mathrm{Be}$}

Finally we present some preliminary results from a study of the ${ }^{3} \mathrm{He}(\alpha, \gamma)^{7} \mathrm{Be}$ reaction. The structure of the nuclei involved in this reaction were obtained using the CD-Bonn $2000 \mathrm{NN}$ interaction in NCSM model spaces up to $10 \hbar \Omega$. Calculations in even larger model spaces will be attempted. Woods-Saxon potential solutions were fitted to the $a b$ initio cluster overlaps in the 0-3.6 $\mathrm{fm}$ range. The scattering states were obtained from a potential model that reproduces experimental phase shifts. The calculated $S$-factor is shown in Fig. 2(c). However, we observe a clear trend of increasing $S$-factor with increasing model space and we stress that convergence tests are still underway. 


\section{Conclusion}

In this paper we have shown that we have the tools and techniques to study strongly interacting, fermionic quantum many-body systems. The main focus has been to demonstrate some of the first attempts of combining $a b$ initio nuclear structure information with the modeling of low-energy reactions. We have also indicated that there are dedicated efforts to achieve a truly fundamental description of nuclear reactions.

\section{Acknowledgments}

This work was partly performed under the auspices of the U. S. Department of Energy by the University of California, Lawrence Livermore National Laboratory under contract No.

W-7405-Eng-48. Support from the LDRD contract No. 04-ERD-058, and from U.S. Department of Energy, Office of Science, (Work Proposal Number SCW0498) is acknowledged.

\section{References}

[1] P. Navrátil, J. P. Vary, and B. R. Barrett, Phys. Rev. C 62, 054311 (2000).

[2] P. Navrátil, Phys. Rev. C 70, 054324 (2004).

[3] C. Forssén, P. Navrátil, W. E. Ormand, and E. Caurier, Phys. Rev. C 71, 044312 (2005).

[4] T. Rauscher, J. Applegate, J. Cowan, F.-K. Thielemann, and M. Wiescher, Astrophy. J. 429, 499 (1994).

[5] A. Mengoni, T. Otsuka, T. Nakamura, and M. Ishihara, Nucl. Phys. A 621, 323 (1997).

[6] C. Forssén, P. Navrátil, W. E. Ormand, and E. Caurier, Manuscript i n preparation.

[7] P. Navrátil, C. A. Bertulani, and E. Caurier, Phys. Rev. C 73, 065801 (2006). 